\title{
Noise-Induced Phenomena in the Kaldor Business Cycle Model*
}

\author{
Sou Nobukawa ${ }^{\dagger}$, Ryohei Hashimoto ${ }^{\ddagger}$, Haruhiko Nishimura $^{\S}$, \\ Teruya YAMANishi ${ }^{\ddagger}$ and Masaru CHIBA ${ }^{\ddagger}$
}

\begin{abstract}
The periodic behavior of macroeconomic indicators or business cycles is a common observation in the economic system. In the past, business cycle modeling often involved the use of non-linear economic dynamic theory, general equilibrium theory, and methods to analyze complex systems, such as agent-based modeling and complex network theory. Several studies have shown that crisis and synchronization in business cycles can be modeled using threshold characteristics in the economic systems. In a non-linear system, it is well known that additive noise leads to qualitative transformations in system behavior, such as coherence resonance, stochastic resonance, and noise-induced transitions (referred to as noise-induced phenomena in this study). Recent studies have shown that noise-induced transitions arise in the non-linear economic business cycle model with multiple attractors around the sub-critical Hopf bifurcation. However, the relationship between the threshold characteristics in the economic dynamics and noise-induced phenomena has not been studied. In this study, we focus on the threshold characteristic of the Kaldor business cycle model under the bias of income, and show that coherence resonance and stochastic resonance can be produced as an effect of this threshold characteristic.
\end{abstract}

\section{Introduction}

The periodic behavior of macroeconomic indicators or business cycles, such as the Kitchen cycle, Kondratiev cycle, and Kuznets cycle, has been observed in economic systems[1-3]. Since the 1920s, business cycle modeling has been used to formulate and evaluate economic policies. The earliest approach was based on non-linear economic dynamic theory, in which the major elements of a business cycle are modeled using bifurcation theory[4,5]. Several studies have also used general equilibrium theory[6-10] and methods for analyzing complex systems, such as agent-based modeling and complex network theory[11-14]. Focusing on threshold characteristics in economic systems, that is, external inputs over certain levels induce transition of the system state, several studies have also shown that crisis and synchronization in business cycles can be well-modeled $[15,16]$.

* Manuscript Received Date: November 25, 2016

$\dagger$ Department of Computer Science, Chiba Institute of Technology; 2-17-1 Tsudanuma, Narashino, Chiba 275-0016, JAPAN

¥ Department of Management Information Science, Fukui University of Technology; 3-6-1 Gakuen, Fukui city, Fukui 910-8505, JAPAN

$\S$ Graduate School of Applied Informatics, University of Hyogo; 7-1-28 Chuo-ku, Kobe, Hyogo 650-8588, JAPAN

Key Words: coherence resonance, stochastic resonance, Kaldor business cycle model, economic dynamics, noise.
However, the business cycle mechanism remains highly controversial.

Qualitative transformations in system behavior due to additive noise are widely observed in many kinds of non-linear systems, such as electrical circuits and biological systems[17-20]. (These phenomena, which include coherence resonance, stochastic resonance, and noise-induced transitions, are called noiseinduced phenomena in this study.) Recent studies have examined the effects of additive noise on business cycles based on non-linear economic dynamic theory[21,22]. Especially, Bashkirtseva et al. have shown that noise-induced transitions[17] arise in nonlinear economic dynamic models, such as the Kaldor business cycle model and the Goodwin business cycle model, with multiple attractors around the subcritical Hopf bifurcation $[23,24]$. These studies analyze the influence of stochastic fluctuations on the business cycle from the perspective of noise-induced phenomena. In addition, the relationship between the threshold characteristics in the economic dynamics and the noise-induced phenomena has not been studied.

Previously, we focused on the threshold characteristic of the Kaldor business cycle model under income bias and demonstrated that coherence resonance[19,25], in which additive noise with appropriate strength makes the system behavior coherent, can be produced as an effect of this threshold characteristic[26]. In this study, based on our research outcomes, we evaluate this coherence resonance and confirm that stochastic resonance[19], in which the appropriate noise en- 
hances the response against the weak input signal, can arise in this system.

\section{Model and Methods}

\subsection{The Kaldor Business Cycle Model}

Kaldor proposed a business cycle model focusing on the dynamics of income $Y$ and capital stock $K$, and the dependence of investment $I$ and saving $S$ on $Y$ and $K$ as follows[4]:

$$
\begin{aligned}
& \dot{Y}(t)=\alpha(I(Y(t), K(t))-S(Y(t)), \\
& \dot{K}(t)=I(Y(t), K(t))-q K(t) .
\end{aligned}
$$

Here, $\alpha$ is the adjustment coefficient in the goods market and $q$ is the depreciation rate of capital stock, respectively. Functions of $S$ and $I$ are given by

$$
\begin{aligned}
& S(Y(t))=p Y(t), \\
& I(Y, K)=p u+r\left(\frac{p u}{q}-K\right)+f(Y-u), \\
& f(x)=\frac{1}{1+\exp (-4 x)}-0.5,
\end{aligned}
$$

where $\left(u, \frac{p u}{q}\right)$ corresponds to a fixed point $(Y, K)$, and $r$ is an adjustment cost. In our simulation, we use $(p, q, r, u)=(0.3,0.2,1.0,3.0)[22]$.

To evaluate the system behavior under an income bias, weak signal, and noise, we consider $b$ as the bias term of $\dot{Y}$, a weak sinusoidal signal $I_{\text {ext }}(t)=$ $A \sin 2 \pi f_{S} t$, and a Gaussian white noise $D \xi(t)(<$ $\xi(t)>=0,<\xi(t), \xi\left(t^{\prime}\right)>=\delta_{t t^{\prime}},<\cdot>$ indicates average in $t$ ) for Eq. (1) as follows:

$$
\begin{aligned}
& \dot{Y}(t)=\alpha(I(Y(t), K(t))-S(Y(t))+b) \\
& +I_{\text {ext }}(t)+D \xi(t) .
\end{aligned}
$$

Here, $I_{\text {ext }}(t)$ and $D \xi(t)$ are typical examples of external influence, such as government intervention and global factors [5]. The initial condition of $(Y(t), K(t))$ is set to $(Y(0), K(0))=(1.0,1.0)$.

\subsection{Index for Evaluation}

To measure periodicity of income $Y(t)$, we adopt the correlation time[25]

$$
\begin{aligned}
& \tau_{c}=\int_{0}^{\infty} C_{y y}(\tau)^{2} d \tau, \\
& C_{y y}(\tau) \\
& =\frac{<(Y(t+\tau)-<Y(t)>)(Y(t)-<Y(t)>)>}{<(Y(t)-<Y(t)>)^{2}>} .
\end{aligned}
$$

Here, $C_{y y}(\tau)$ indicates the auto-correlation of $Y(t)$ on time delay $\tau . \tau_{c} \approx 0$ and $\tau_{c} \gg 0$ correspond to cases in which the time series of $Y(t)$ becomes irregular and periodic, respectively.

The response of $Y(t)$ against the signal $I_{\text {ext }}(t)$ is measured by the signal to noise ratio:

$$
S N R=10 \log _{10} \frac{P_{S}}{P_{N}},
$$

$$
\begin{aligned}
& P_{S}=\frac{1}{2 \Delta f_{S}} \int_{f_{S}-\Delta f_{S}}^{f_{S}+\Delta f_{S}} P(f) d f \\
& P_{N}=\frac{1}{2\left(\Delta f_{N}-\Delta f_{S}\right)}\left(\int_{f_{S}-\Delta f_{N}}^{f_{S}-\Delta f_{S}} P(f) d f\right. \\
& \left.+\int_{f_{S}+\Delta f_{S}}^{f_{S}+\Delta f_{N}} P(f) d f\right) .
\end{aligned}
$$

Here, $P(f)$ indicates the power spectrum of the binarized time series of $Y(t)$ with the threshold of $Y(t)=$ 3.0. $P_{S}$ and $P_{N}$ indicate the powers of the signal component and the noise component, respectively. In our simulation, we set $\Delta f_{S}=2.04 \times 10^{-4}, \Delta f_{N}=0.03$.

For the bifurcation analysis in the system, we utilize the eigenvalues $l_{j}(j=1,2)$ of the Jacobian $J$ for $(\dot{Y}, \dot{K})$ around the fixed point $\left(Y_{f}, K_{f}\right)$ as follows:

$$
J=\left(\begin{array}{cc}
\alpha\left(4 e^{-4 u_{0}} /\left(\left(1+e^{-4 u_{0}}\right)^{2}-p\right)\right. & -\alpha r \\
4 e^{-4 u_{0}} /\left(1+e^{-4 u_{0}}\right)^{2} & -r-q
\end{array}\right),
$$

where $u_{0}$ indicates $Y_{f}-u$.

\section{Results and Evaluations}

\subsection{System Behavior of the Kaldor Busi- ness Cycle Model}

At first, we demonstrate the appearance of a limit cycle in the absence of bias, noise, and signal $(b=$ $0, D=0, A=0)$. Fig. 1 shows the maximum real part of eigenvalue $l_{j}$ given by Eq.(12) around the fixed point $\left(u, \frac{p u}{q}\right): \max _{j} \operatorname{Re}\left(l_{j}\right)$. In $0 \leq \alpha \lesssim 1.7$, the fixed point is stable $\left(\max _{j} \operatorname{Re}\left(l_{j}\right)<0\right)$. In this case, the orbit of $(Y, K)$ stays at $\left(u, \frac{p u}{q}\right)$, which is the intersection between the $Y$-nullcline $(\dot{Y}=0)$ and the $K$-nullcline $(\dot{K}=0)$ as shown in Fig. 2 (a). In $1.7 \lesssim \alpha \lesssim 3.0$ (Fig.1), the fixed point becomes unstable $\left(\max _{j} \operatorname{Re}\left(l_{j}\right)>0\right)$. Furthermore, considering the imaginary parts of $l_{1}$ and $l_{2}$, these complex conjugates intersect with the real axis at $\alpha \approx 1.7$; this bifurcation is specified as the Hopf bifurcation. This bifurcation produces the limit cycle along the vector field, as shown in Fig.2 (b).

\subsection{Dependence of System Behavior on Bias of Income}

Next, we investigate the influence of the bias of income $b$ on the $Y$-nullcline and the $K$-nullcline in a noise/signal-free condition. Fig. 3 shows the vector field and the nullclines in $b=0((\mathrm{a}))$ and $b=-0.1$ cases $((\mathrm{b}))$. For $b=0$, the nullclines intersect at $(Y, K) \approx$ $(3.0,4.5)$. If we apply the perturbation to this system, the orbit of $(Y, K)$ moves from the fixed point, according to the strength of this perturbation. On the other hand, for $b=-0.1$, the intersection moves to $(Y, K) \approx(2.5,4.2)$. If the perturbation above a certain level is input, the orbit of $(Y, K)$ is drawn to the flow in $K \lesssim 4.2$ and reaches the region with large values of $Y(\approx 4.0)$. This system behavior is the threshold characteristic.

Furthermore, the dependence of this threshold characteristic on the parameters $\alpha$ and $b$ are evaluated. 


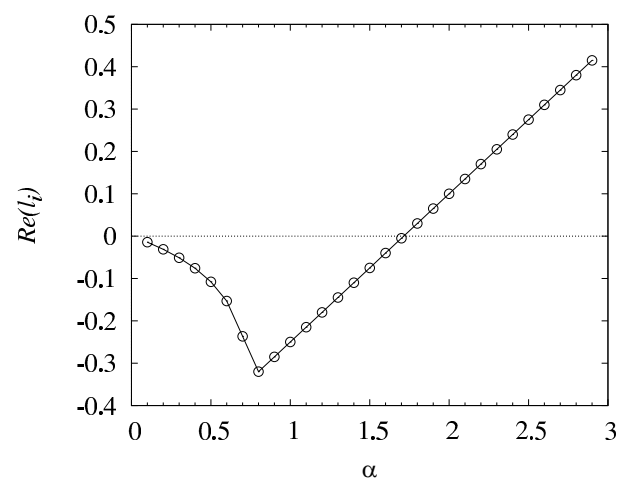

Fig. 1 Maximum real part of eigenvalue $\max _{j} \operatorname{Re}\left(l_{j}\right)$ of Jacobian for $(\dot{Y}, \dot{K})$ at fixed point $\left(u, \frac{p u}{q}\right)$ between gross product $Y(t)$ and capital stock $K(t)$ as the function of the adjustment coefficient $\alpha$. $(p=0.3, r=1.0, q=0.2, u=3.0, A=0, b=0, D=0)$

(a)

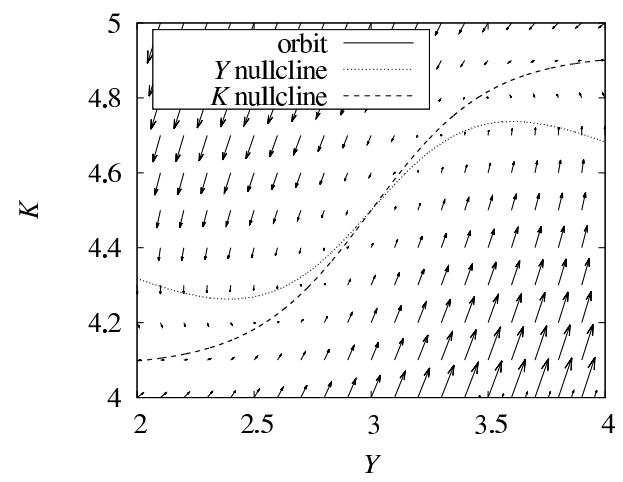

(b)

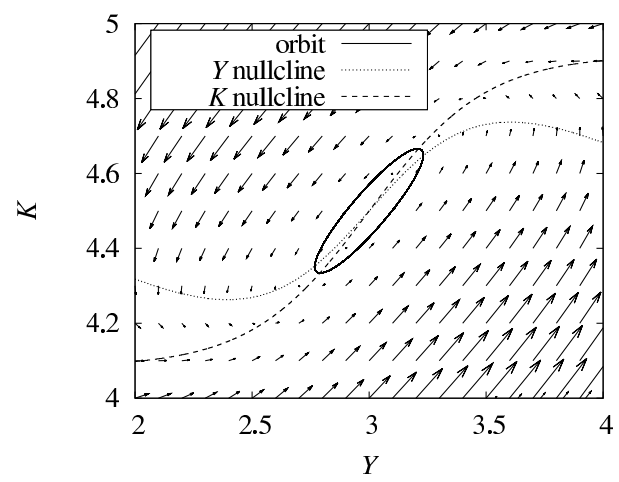

Fig. 2 Orbit of $(Y(t), K(t)), \quad Y$-nullcline $(\dot{Y}=0), \quad K$ nullcline $(\dot{K}=0)$, and vector field $(\dot{Y}, \dot{K})$ on phase plane $Y-K$. (a) $\alpha=0.8$. (b) $\alpha=1.8$. $(p=0.3, r=$ $1.0, q=0.2, u=3.0, A=0, b=0, D=0)$

Fig. 4 shows the maximum and minimum values of $Y(t)$ as a function of $b$ in the $\alpha=2.0,5.0,8.0$ cases. In the $\alpha=2.0$ case, $Y(t)$ increases accompanied by $b$ in $-0.2 \leq b \lesssim-0.02$ and begins to oscillate from $b \approx-0.02$. In the $\alpha=5.0,8.0$ cases, the oscillations begin from $b \approx-0.08,-0.09$, respectively. The amplitude of oscillation becomes larger with increasing $\alpha$.

Additionally, we examine the bifurcation as a func- (a)

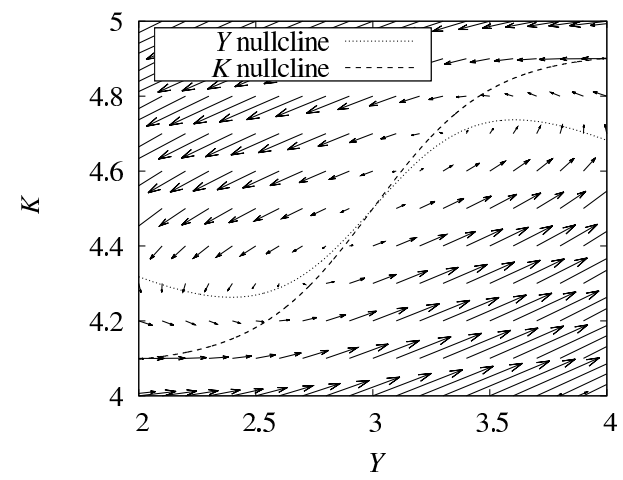

(b)

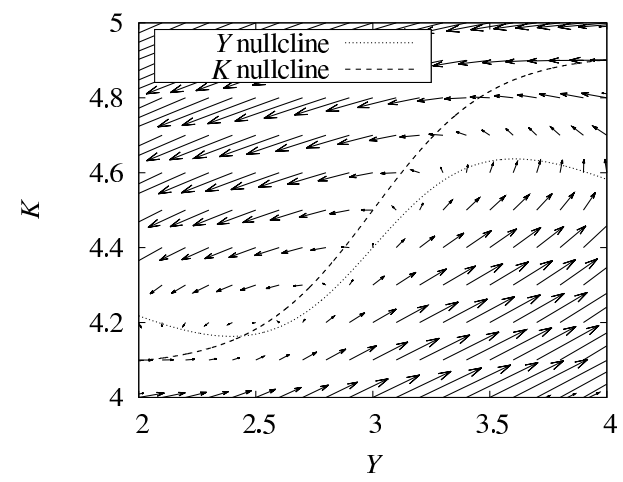

Fig. 3 Orbit of $(Y(t), K(t)), \quad Y$-nullcline $(\dot{Y}=0), \quad K$ nullcline $(\dot{K}=0)$, and vector field $(\dot{Y}, \dot{K})$ on phase plane $Y-K$. (a) $b=0$. (b) $b=-0.1$. $(p=0.3, r=$ $1.0, q=0.2, u=3.0, \alpha=5.0, A=0, D=0)$

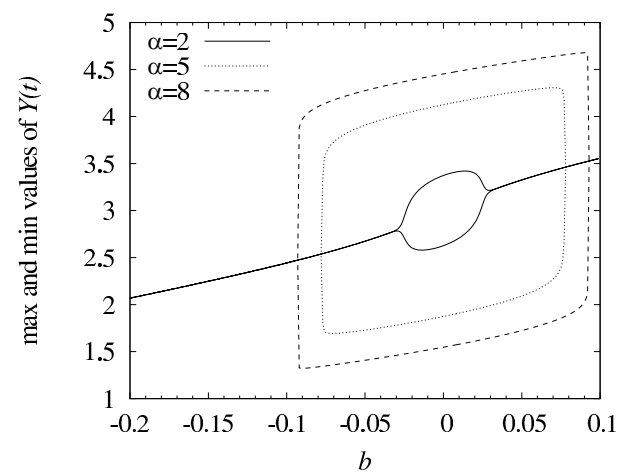

Fig. 4 Dependence of maximum and minimum values of gross product $Y(t)$ on parameter $b . \quad(p=0.3, r=$ $1.0, q=0.2, u=3.0, A=0, D=0)$

tion of parameters $\alpha$ and $b$ by $l_{j}$. The bifurcation diagram, as shown in Fig. 5 indicates the regions where the fixed point becomes stable (gray region) and where the limit cycle arises around the unstable fixed point (white region) through the Hopf bifurcation (red dashed line). Further, the stable fixed point and limit cycle co-exist at the outer edge of the white region given by the green dots. These results specify this Hopf bifurcation as a sub-critical Hopf bifurcation. 


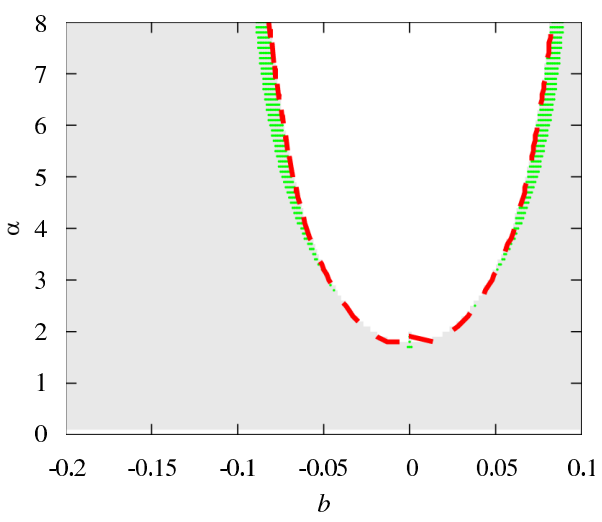

Fig. 5 Bifurcation diagram by eigenvalues of Jacobian $J$ for $(\dot{Y}, \dot{K})$ around fixed point. Gray region: fixed point becomes stable. White region: limit cycle arises and fixed point becomes unstable. Green points: stable fixed point and limit cycle co-exist. Red dashed line: sub-critical Hopf bifurcation. $(p=0.3, r=1.0, q=0.2, u=3.0, A=0, D=0)$

\subsection{Coherence Resonance of the Kaldor Business Cycle Model}

In this section, we evaluate the influence of noise on system behavior in three types of conditions examined in the previous section. These are the conditions for the existence of a stable fixed point, limit cycle, and the co-existence of a stable fixed point and a limit cycle.

Fig. 6 shows the time series of $Y(t)$ (left) and its cross correlation $C_{y y}(\tau)$ given by Eq.(8) (right) in case of $D=5.0 \times 10^{-3}, 0.1,0.3,1.0,5.0$ under the condition for the existence of a stable fixed point $(\alpha=$ $5.0, b=-0.1)$. At $D=5.0 \times 10^{-3}$ (a), $0.1(\mathrm{~b}), Y(t)$ stays around the fixed point. However, $Y(t)$ exhibits oscillation with the period of approximate 10 at $D=$ 0.3 (c). In condition of stronger noise strength $(D=$ $1.0(\mathrm{~d}), 5.0(\mathrm{e}))$, the random behavior of $Y(t)$ becomes dominant. Owing to this tendency of $Y(t)$, it is hard to decay the oscillation of $C_{y y}(\tau)$ at $D=0.1$ (b) in comparison with other noise strength cases.

As shown in the open triangles of Fig. 7 (a), the dependence of $\tau_{c}$ given by Eq.(7) that corresponds to this behavior at $b=-0.1$ on noise strength $D$ is demonstrated. $\tau_{c}$ indicates approximately 0.9 in $5.0 \times$ $10^{-3} \leq D \lesssim 5.0 \times 10^{-2}$; however, $\tau_{c}$ rises with increasing $D$. Then, at $D \approx 0.3, \tau_{c}$ achieves its peak value $\left(\tau_{c} \approx 1.6\right)$. That is, coherence resonance arises in the Kaldor business cycle model with the bias of income. In condition of $b=-0.15,-0.2$ indicated by the open circle and square, the fixed point moves away from the threshold in the negative direction. Therefore, it needs stronger noise strength to achieve the peak of $\tau_{c}$ and deteriorates the peak value. Fig. 7 (b) shows the existence of a limit cycle $(b=-0.05)$ and co-existence of stable fixed point and limit cycle $(b=$ $-0.072)$. Note that the location of the initial condition $(Y(0), K(0))=(1.0,1.0)$ is outside the orbit of limit cycle in $(b=-0.072)$. In both cases the val- (a)
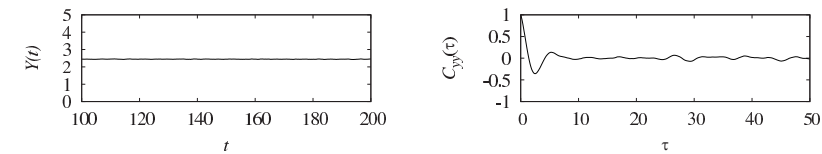

(b)
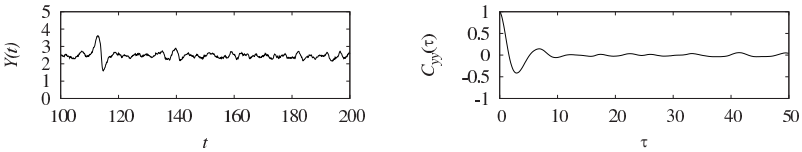

(c)
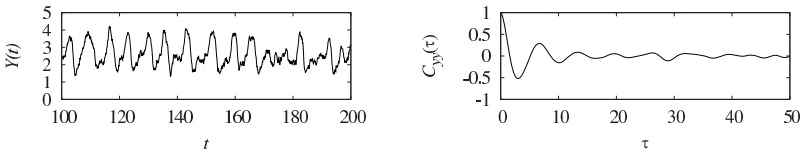

(d)
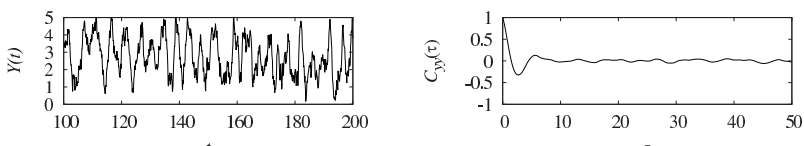

(e)
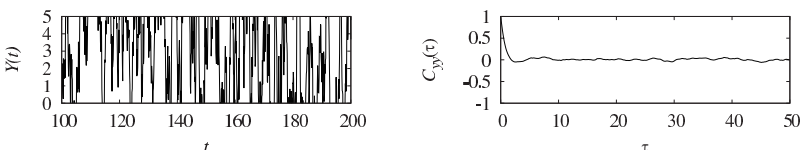

Fig. 6 Time series of gross product $Y(t)$ (left) and its cross correlation $C_{y y}(\tau)$ (right). (a) $D=5 \times 10^{-3}$. (b) $D=0.1 . \quad$ (c) $D=0.3 . \quad$ (d) $D=1.0$. (e) $D=5.0$. $(p=0.3, r=1.0, q=0.2, u=3.0, \alpha=5.0, b=$ $-0.1, A=0)$

ues of $\tau_{c}$ decreases according to the noise strength $D$ due to disturbing autonomous oscillations; therefore, the peak of $\tau_{c}$ is not confirmed. In the positive bias $(b>0)$ cases of the existence of a stable fixed point (see Fig. 7 (c)), limit cycle $(b=0.05$ in Fig. $7(d))$ and the co-existence of stable fixed point and limit cycle $(b=0.072$ in Fig. $7(d))$, we confirm the same tendency with negative bias cases due to the symmetric structure of $\dot{Y}$ and $\dot{K}$ against $\left(u, \frac{p u}{q}\right)$.

\subsection{Stochastic Resonance of Kaldor Busi- ness Cycle Model}

In this section, the response against the input signal $I_{\text {ext }}(t)$ under the noise is evaluated in the three types of conditions mentioned above. Fig. 8 shows the time series of $Y(t)$ (left) and its power spectrum $P(f)$ (right) in the case of $D=4.0 \times 10^{-3}, 0.06,0.1$, $0.5,5.0$ under the existence of a stable fixed point $(\alpha=5.0, b=-0.1)$ and applying $I_{\text {ext }}(t)\left(A=0.1, f_{S}=\right.$ 0.1 ). At $D=4.0 \times 10^{-3}$ (a), $Y(t)$ stays around the fixed point. However, the oscillation with the period of approximate $1 / f_{S} \approx 10$ appears at $D=0.06$ (b) and $D=0.1(\mathrm{c})$. This periodicity can be also observed by the peak of the power spectrum $P(f)$ at $f \approx f_{S}=0.1$. This oscillation disappears when increasing noise strength further, as shown in Figs. 8 (d) and (e).

The dependence of $S N R$ given by Eq.(9) corresponding to this behavior at $b=-0.1$ on noise strength $D$ is demonstrated by the open triangles of Fig. 9 (a). 
(a)

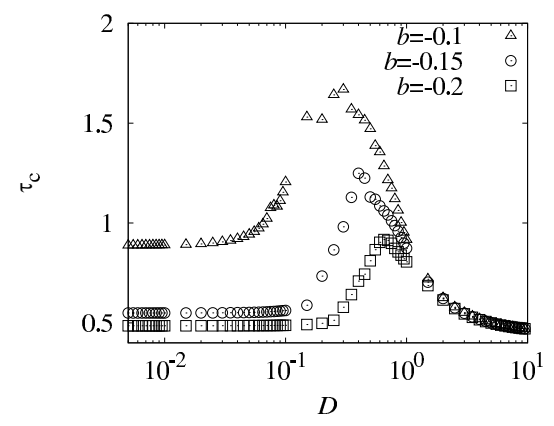

(c)

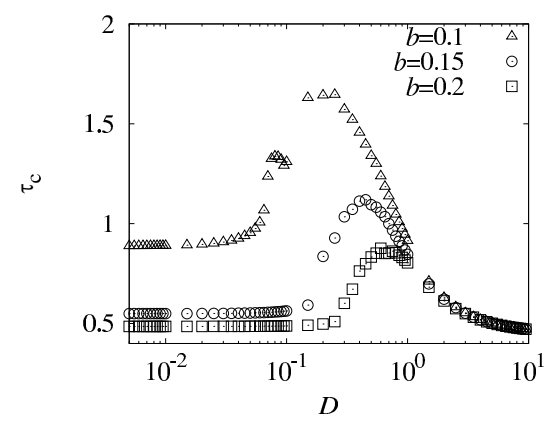

(b)

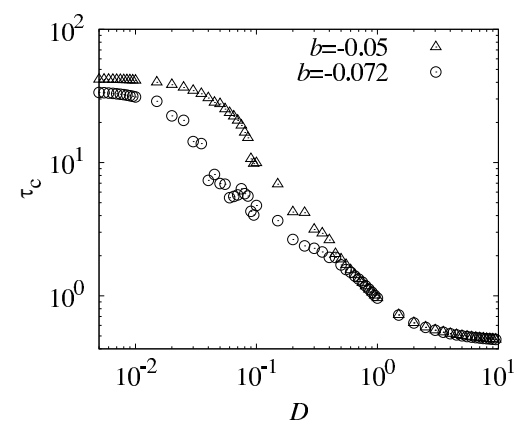

(d)

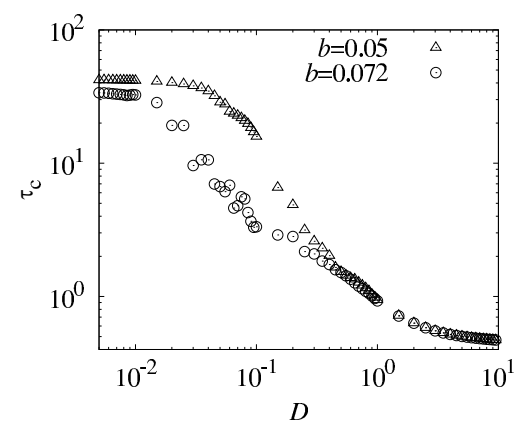

Fig. 7 Dependence of characteristic correlation time $\tau_{c}$ on noise strength $D$. (a) Condition for the existence of stable fixed point in negative bias. (b) Condition for the existence of limit cycle $(b=-0.05)$ and for the co-existence of stable fixed point and limit cycle $(b=-0.072)$ in negative bias. (c) Condition for the existence of stable fixed point in positive bias. (d) Condition for the existence of limit cycle $(b=0.05)$ and for the co-existence of stable fixed point and limit cycle $(b=0.072)$ in positive bias. $(p=0.3, r=1.0, q=0.2, u=3.0, \alpha=5.0, A=0)$

(a)

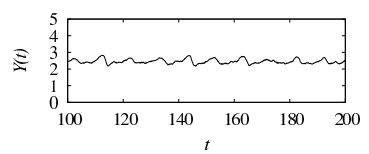

(b)

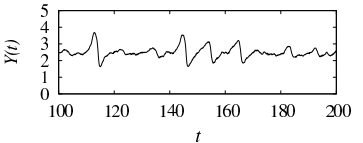

(c)

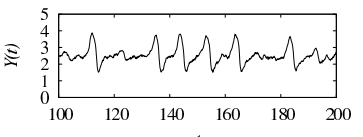

(d)

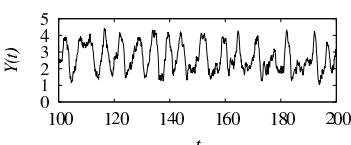

(e)

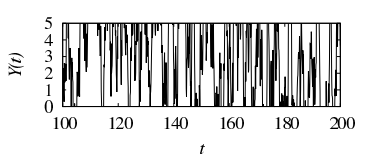

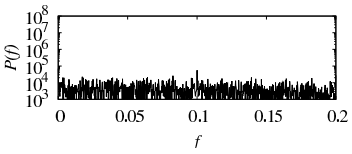
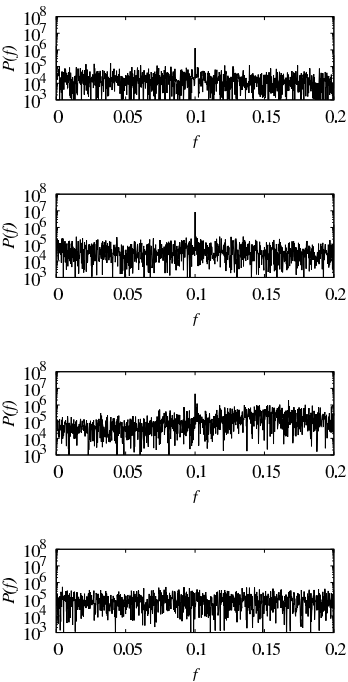

Fig. 8 Time series of gross product $Y(t)$ (left) and the power spectrum $P(f)$ of the binarized time series of $Y(t)$ (right). (a) $D=4.0 \times 10^{-3}$. (b) $D=0.06$. (c) $D=0.1$. (d) $D=0.5$. (e) $D=5.0$. $(p=0.3, r=$ $1.0, q=0.2, u=3.0, \alpha=5.0, b=-0.1, A=0.1, f_{S}=$ $0.1)$

$S N R$ indicates approximately 0 at $D=3.0 \times 10^{-2}$;

however, $S N R$ rises with increasing $D$. Then, at $D \approx 0.5, S N R$ achieves its peak value $(S N R \approx 22)$. We can interpret this enhancement of signal response by noise with the appropriate strength as stochastic resonance. In conditions of $b=-0.15$ (open circle) and -0.2 (open square), the peak of $S N R$ decreases due to the shift in the fixed point in the negative direction, as in the coherence resonance case. Fig. 9 (b) shows the cases with existence of a limit cycle $(b=-0.05)$ and co-existence of the stable fixed point and limit cycle $(b=-0.072)$. Note that the location of $(Y(0), K(0))$ is outside the orbit of limit cycle in the case of $(b=-0.072)$, as well as the coherence resonance case in section 3.3. In these cases, the peak of $S N R$ does not exist as it does in the coherence resonance case. In the positive bias $(b>0)$ cases of the existence of a stable fixed point, we confirm the same tendency with negative bias cases due to the symmetric structure of $\dot{Y}$ and $\dot{K}$, as shown in Fig.9 (c) and (d).

We also examine dependence on signal strength $A$ in stochastic resonance. Fig. 10 shows the dependence of $A$ and $D$ on $S N R$ under the condition of fixing $b=-0.1$. In $10^{-3} \leq A \lesssim 10^{-2}$, the signal response efficiency is low $(S N R \approx 0)$, but increases accompanied with signal strength $A$, the value of $S N R$ exhibits its peak against the changing value of $D$ at $D \approx 0.1$ in $10^{-2} \lesssim A \leq 0.2$. However, further enhancing signal strength $(A \gtrsim 0.2)$, decreases $S N R$ according to the noise strength $D$; therefore, we cannot confirm the 
(a)

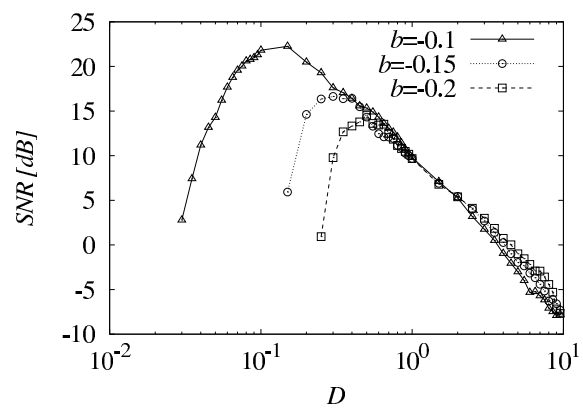

(c)

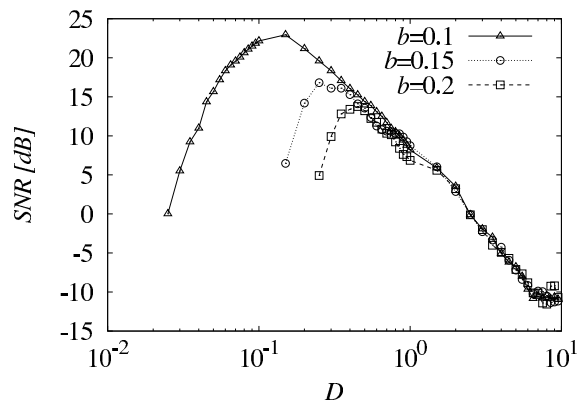

(b)

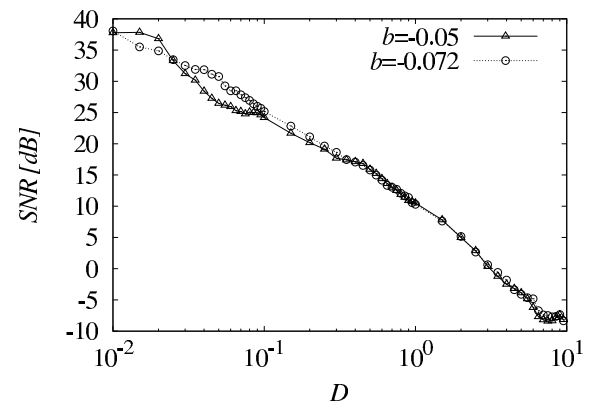

(d)

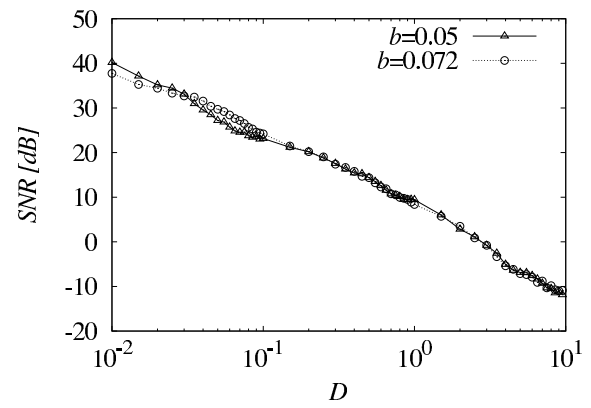

Fig. 9 Dependence of $S N R$ on noise strength $D$. (a) Condition for the existence of a stable fixed point in negative bias. (b) Condition for the existence of a limit cycle $(b=-0.05)$ and for the co-existence of stable fixed point and limit cycle $(b=-0.072)$ in negative bias. (c) Condition for the existence of a stable fixed point in positive bias. (d) Condition for the existence of limit cycle $(b=0.05)$ and for the co-existence of stable fixed point and limit cycle $(b=0.072)$ in positive bias. $\left(p=0.3, r=1.0, q=0.2, u=3.0, \alpha=5.0, A=0.1, f_{S}=0.1\right)$

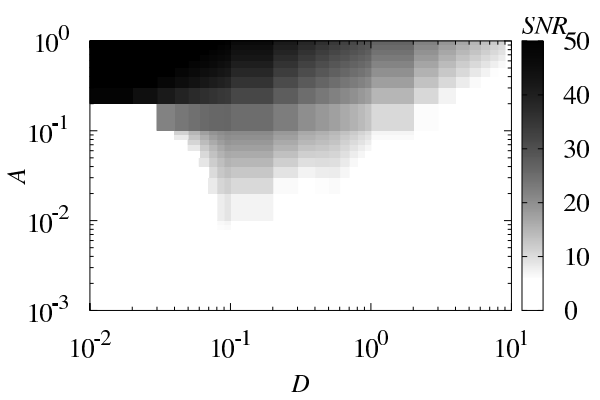

Fig. 10 Dependence of $S N R$ on noise strength $D$ and signal strength $A . \quad(p=0.3, r=1.0, q=0.2, u=$ $\left.3.0, \alpha=5.0, b=-0.1, f_{S}=0.1\right)$

peak of $S N R$.

Next, we evaluate dependence on the signal frequency in stochastic resonance under the condition ( $A=0.1, D=0.07, b=-0.1)$. As shown in Fig. 11, the signal frequency $f_{S}$ dependence of $S N R$ peaks at $f_{S} \approx 0.15$. Thus, stochastic resonance has the resonance frequency.

\section{Conclusion}

In this study, we examined system behavior in the Kaldor business cycle model under the bias of income. We found that a negative/positive bias led to the threshold characteristic of the system. In addition, through bifurcation analysis, we confirmed three kinds of distinctive parameter regions. The first was the existence of a stable fixed point. The second was

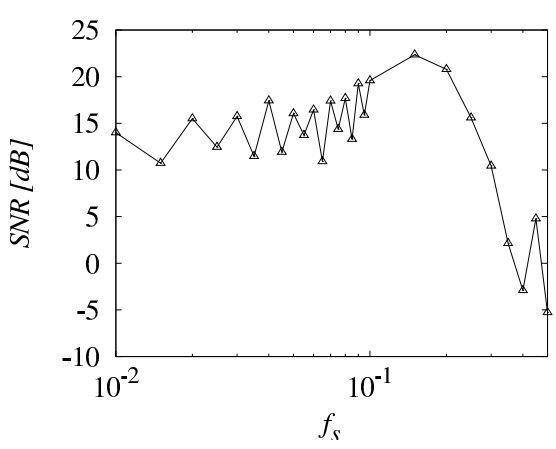

Fig. 11 Dependence of $S N R$ on signal frequency $f_{S}$. ( $p=$ $0.3, r=1.0, q=0.2, u=3.0, \alpha=5.0, b=-0.1, A=$ $0.1, D=0.07)$

the existence of a limit cycle and the third was the co-existence of a stable fixed point and a limit cycle. Furthermore, we investigated coherence resonance in these regions using $\tau_{c}$. Consequently, we confirmed that coherence resonance is induced by noise with an appropriate strength in the first region. However, in other regions, the additive noise disturbs the orbit of autonomous oscillations based on its strength. Finally, under weak input signal, we evaluated stochastic resonance in the above three regions using $S N R$. The result showed that stochastic resonance is produced by noise with an appropriate strength, and a signal with weak strength and specific frequency only in the first region, as in the coherence resonance case. That is, the business cycle rests under the condition 
of decreasing/increasing income; however, the additive noise induces regeneration in the business cycle due to coherence resonance and stochastic resonance. If the business cycle exists in the noise-free condition, the noise only perturbs the business cycle based on its strength.

In future studies, we plan to examine noise-induced phenomena in more realistic models for dynamic economies to evaluate the economic validity of the mechanisms dealt with in this paper.

\section{References}

[1] J. Kitchin: Cycles and trends in economic factors; The Review of Economic Statistics, pp. 10-16 (1923)

[2] N. D. Kondratiev: The major economic cycles; Voprosy Konjunktury, Vol. 1, No. 1, pp. 28-79 (1925)

[3] S. S. Kuznets, et al.: Secular Movements in Production and Prices, Houghton Mifflin (1930)

[4] N. Kaldor: A model of the trade cycle; The Economic Journal, pp. 78-92 (1940)

[5] H.-W. Lorenz: Nonlinear Dynamical Economics and Chaotic Motion, Springer (1993)

[6] R. E. Lucas: Understanding business cycles; Carnegie-Rochester Conference Series on Public Policy, Vol. 5, North-Holland, pp. 7-29 (1977)

[7] F. E. Kydland and E. C. Prescott: Time to build and aggregate fluctuations; Econometrica: Journal of the Econometric Society, pp. 1345-1370 (1982)

[8] J. B. Long Jr and C. I. Plosser: Real business cycles; The Journal of Political Economy, pp. 39-69 (1983)

[9] C. I. Plosser: Understanding real business cycles; The Journal of Economic Perspectives, Vol. 3, No. 3, pp. 51-77 (1989)

[10] J.-P. Danthine and A. Kurmann: Fair wages in a new keynesian model of the business cycle; Review of Economic Dynamics, Vol. 7, No. 1, pp. 107-142 (2004)

[11] L. Tesfatsion: Agent-based computational economics: Modeling economies as complex adaptive systems; Information Sciences, Vol. 149, No. 4, pp. 262-268 (2003)

[12] M. E. Newman: The structure and function of complex networks; SIAM Review, Vol. 45, No. 2, pp. 167256 (2003)

[13] T. Preis, J. J. Schneider and H. E. Stanley: Switching processes in financial markets; Proceedings of the National Academy of Sciences, Vol. 108, No. 19, pp. 7674-7678 (2011)

[14] Y. Mishchenko and A. R. H. Montoya: Oscillations in rational economies; PloS One, Vol. 9, No. 2, e87820 (2014)

[15] S. Dées and N. Zorell: Business cycle synchronisation: Disentangling trade and financial linkages; Open Economies Review, Vol. 23, No. 4, pp. 623-643 (2012)

[16] M. de Carvalho, K. Turkman and A. Rua: Dynamic threshold modelling and the US business cycle; Journal of the Royal Statistical Society: Series C (Applied Statistics), Vol. 62, No. 4, pp. 535-550 (2013)

[17] W. Horsthemke: Noise Induced Transitions, Springer (1984)

[18] F. Moss and K. Wiesenfeld: The benefits of back- ground noise; Scientific American, Vol. 273, pp. 6669 (1995)

[19] L. Gammaitoni, P. Hänggi, P. Jung and F. Marchesoni: Stochastic resonance; Reviews of Modern Physics, Vol. 70, No. 1, pp. 223-287 (1998)

[20] P. Hänggi: Stochastic resonance in biology how noise can enhance detection of weak signals and help improve biological information processing; ChemPhysChem, Vol. 3, No. 3, pp. 285-290 (2002)

[21] D. Huang, H. Wang and Y. Yi: Bifurcations in a stochastic business cycle model; International Journal of Bifurcation and Chaos, Vol. 20, No. 12, pp. 4111-4118 (2010)

[22] G. Mircea, M. Neamtu and D. Opris: The Kaldor stochastic model of business cycle; Nonlinear Anal Model Control, Vol. 16, No. 2, pp. 191-205 (2011)

[23] I. Bashkirtseva, T. Ryazanova and L. Ryashko: Confidence domains in the analysis of noise-induced transition to chaos for goodwin model of business cycles; International Journal of Bifurcation and Chaos, Vol. 24, No. 81440020 (2014)

[24] I. Bashkirtseva, T. Ryazanova and L. Ryashko: Analysis of dynamic regimes in stochastically forced kaldor model; Chaos, Solitons \& Fractals, Vol. 79, pp. 96-104 (2015)

[25] A. S. Pikovsky and J. Kurths: Coherence resonance in a noise-driven excitable system; Physical Review Letters, Vol. 78, No. 5, pp. 775-778 (1997)

[26] S. Nobukawa, R. Hashimoto, H. Nishimura, T. Yamanishi and M. Chiba: Analysis of coherence resonance in Kaldor-Kalecki business cycle model; Proceedings of Joint 8th International Conference on Soft Computing and Intelligent Systems (SCIS) and 17th International Symposium on Advanced Intelligent Systems (ISIS), IEEE, pp. 243-247 (2016)

\section{Authors}

Sou NoBUKaWA (Member)

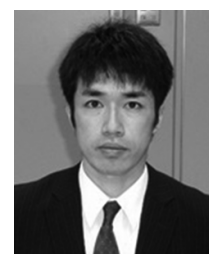

graduated from the Department of Physics and Earth Sciences University of Ryukyus in 2006, and completed the doctoral program at University of Hyogo and received the Ph.D. degree in 2013. He is an Associate Professor in Department of Computer Science, Chiba Institute of Technology. His research interests include chaos/bifurcation and neural network. $\mathrm{He}$ is a member of IEEE, INNS, IEICE, IPSJ, SICE, ISCIE, and others, and was awarded SICE encouraging prize in 2016

\section{Ryohei Hashimoto}

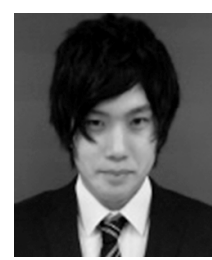

graduated from Fukui University of Technology. His research interests include bifurcation and noise-induced phenomena of non-linear economic dynamical system. He was awarded SICE encouraging prize of Hokuriku chapter in 2015. 
Haruhiko Nishimura (Member)

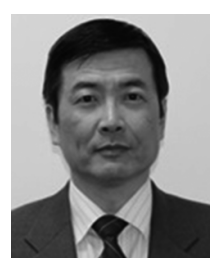

graduated from the Department of Physics, Shizuoka University in 1980, and completed the doctoral program at Kobe University and received the Ph.D. degree in 1985. He is currently a Professor in the Graduate School of Applied Informatics, University of Hyogo. His research field is intelligent systems science by several architectures such as neural networks and complex systems. He is also presently engaged in research on biomedical, healthcare, and high confidence sciences. He is a member of the IEEE, IEICE, IPSJ, ISCIE, JNNS, and others, and was awarded ISCIE paper prize in 2001 and JSKE paper prize in 2010

\section{Teruya YAMANISHI}

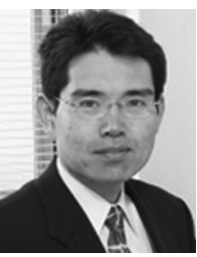

received the Master's degree in educations of science from Kobe University in 1991, and the Ph.D. degree in physics from Kobe University in 1994. He is a Professor at Fukui University of Technology where he studies mathematical information science for the brain, and develops optimization tools on behaviors of the autonomous robots.

\section{Masaru CHIBA}

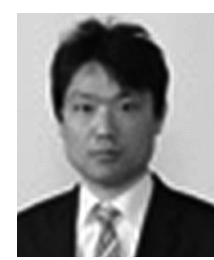

graduated from Faculty of Business Administration, Tokyo University of Science in 2003, and completed the doctoral program at Yokohama National University and received the Ph.D. degree in 2008. He is an Associate Professor in Department of Management and Information Sciences, Fukui University of Technology. His research interests state-space models of financial data. 